\title{
Le protocole EMDR intégratif de traitement de groupe pour les patients atteints de cancer
}

\author{
Ignacio Jarero \\ Lucina Artigas \\ Susana Uribe \\ Laura Evelyn García \\ Fondation latino-américaine et caribéenne pour la recherche sur les traumatismes psychologiques, Mexique
}

\begin{abstract}
L'expérience du cancer est un facteur de stress particulier au sein de l'infrastructure de l'état de stress post-traumatique (ESPT) car cette maladie débilitante implique des facteurs de stress permanents, et elle est à la fois aiguë et potentiellement chronique. Le cancer peut s'accompagner d'un large éventail d'effets négatifs connexes, tels que la détection d'une tumeur, le diagnostic, la gravité de la maladie et le pronostic, l'agressivité des traitements, la mutilation et le dysfonctionnement physique, les effets secondaires du traitement, les perturbations du fonctionnement physique, social et professionnel et, parfois, la récidive et un diagnostic de maladie terminale. Cet article fournit une description détaillée de l'application clinique du protocole intégratif de traitement de groupe de désensibilisation et de retraitement par les mouvements oculaires (EMDR-IGTP) adapté aux patients adolescents et adultes atteints de cancer et vivant avec un stress traumatique continu. Ce protocole administre les huit phases du traitement EMDR individuel à un groupe de patients en utilisant une composante de l'art-thérapie (c'est-à-dire des dessins) et le « butterfly hug » (BH - le " câlin de papillon », une méthode de stimulation bilatérale autoadministrée utilisée pour traiter le matériel traumatique). Une étude antérieure (Jarero et coll., 2015) avait montré qu'après six séances d'EMDR-IGTP, on observait une diminution significative des symptômes d'ESPT liés au diagnostic et au traitement de différents types de cancer chez des femmes adultes. Les effets se maintenaient lors de la séance de suivi, 90 jours plus tard. Dans le présent article, nous discutons de la manière dont on peut se servir de ce protocole pour fournir efficacement un traitement EMDR intensif à de grands groupes de patients, et nous donnons des instructions détaillées pour son utilisation, afin d'aborder l'une des principales dimensions psychologiques du cancer : les réactions de stress traumatique permanentes que connaissent les patients. Un exemple clinique illustre le processus de traitement.
\end{abstract}

Mots-clés : désensibilisation et retraitement par les mouvements oculaires (EMDR) ; protocole EMDR intégratif de traitement de groupe (EMDR-IGTP) ; patients atteints de cancer ; psychothérapie ; état de stress post-traumatique (ESPT) ; traitement EMDR intensif.

\section{$\mathbf{L}$} e cancer est une maladie invalidante potentiellement à la fois aiguë et chronique. Le diagnostic de cancer est un facteur de stress traumatique unique (Andrykowski \& Kangas, 2010) et un large éventail d'effets indésirables connexes peuvent accompagner l'expérience du patient : détection d'une tumeur, diagnostic, gravité de la maladie et pronostic, agressivité des traitements, mutilation et dysfonctionnements physiques, effets secondaires du traitement, perturbations du fonctionnement physique, social et professionnel et, parfois, récidive de la maladie et diagnostic de phase terminale (Jarero et coll., 2015).

This article originally appeared as Jarero, I., Artigas, L., Uribe, S., \& Garcia, L. E. (2016). The EMDR Integrative Group Treatment Protocol for Patients With Cancer, 10(3), 199-207. Translated by François Mousnier-Lompré. 
Les diagnostics d'état de stress post-traumatique (ESPT), de trouble du stress aigu (TSA) et de trouble d'ajustement ne sont pas rares. Kangas, Henry et Bryant (2002) ont passé en revue 13 études concernant l'apparition d'ESPT à la suite d'un diagnostic de cancer ; ils rapportent qu'entre $5 \%$ et $19 \%$ des patients remplissaient les critères du diagnostic d'ESPT. Cet écart correspond à plusieurs facteurs, comme le type, l'évolution et la gravité de la maladie. Dans une autre étude (Kangas, Henry, \& Bryant, 2007), les mêmes chercheurs ont étudié les corrélats du TSA suite à un diagnostic de différents types de cancer (cerveau, gorge et poumon). Un TSA a été diagnostiqué chez $28 \%$ des 82 participants. Environ $21 \%$ des patients atteints du cancer développent ce qu'on appelle des troubles d'ajustement (Muszbek, 2010), qui sont des états psychologiques intermédiaires entre la réaction normale et des signes de trouble mental majeur (American Psychiatric Association, 2013). Certains patients atteints du cancer peuvent souffrir d'un trouble d'ajustement chronique, parce qu'ils sont confrontés à de nombreuses causes de détresse se succédant sans trêve (National Cancer Institute, 2015).

\section{Conceptualisation du traitement}

Nous utilisons le modèle de traitement adaptatif de l'information de Shapiro (2001) dans notre conceptualisation des effets du traumatisme et du rôle de la thérapie de désensibilisation et de retraitement par les mouvements oculaires (EMDR) dans la résolution de souvenirs non traités, considérés comme étant à l'origine de symptômes pathologiques. Nous avons discuté précédemment de la nature des événements traumatiques aigus permanents, pour lesquels il n'y a pas de période de sécurité post-traumatique (Jarero, Artigas et Luber, 2011 ; Jarero \& Uribe, 2011, 2012). Par exemple, un patient ayant reçu un diagnostic de cancer il y a 20 mois pourrait être conceptualisé comme se trouvant dans une situation de traumatisme grave car, à la suite du diagnostic, le patient a traversé un continuum d'événements stressants : analyses physiquement éprouvantes, traitements agressifs entraînant des effets secondaires, chirurgie et mutilations d'organes, dysfonctionnements corporels, etc. Ce type de stress traumatique aigu et continu nécessite une autre approche de traitement EMDR que celui généralement prévu pour l'état de stress post-traumatique ou le TSA. La consolidation des souvenirs traumatiques est rendue impossible. Au lieu de cela, le réseau de souvenirs du patient reste dans un état d'excitation permanent, se développant à chaque événement stressant ultérieur dans ce continuum (comme les ondes se propageant à la surface d'un étang lorsqu'on y jette une pierre). Il se crée alors un réseau mnésique traumatique cumulatif (Jarero, Amaya, Givaudan et Miranda, 2013) s'étendant jusqu'au moment présent, et qui est souvent à l'origine d'inquiétudes inadaptées et/ou catastrophiques concernant l'avenir (Logie \& de Jongh, 2014). Il est essentiel que le processus thérapeutique identifie et cible chaque incident relié aux autres au sein de ce continuum.

\section{La thérapie EMDR}

Selon l'Organisation mondiale de la santé (OMS, 2013), la thérapie cognitive-comportementale (TCC) centrée sur les traumatismes et la thérapie EMDR sont les seules psychothérapies recommandées pour le traitement des enfants, des adolescents et des adultes atteints d'ESPT. La thérapie EMDR est une approche intrinsèquement axée sur le patient ; elle met l'accent sur sa capacité innée à guérir, à travers ce qu'on suppose être l'activation d'un mécanisme physiologique de traitement adapté de l'information qui demande une "intrusion clinique minimale " (Shapiro, 2001, p. 18). L'EMDR est une procédure standardisée en huit étapes, développée pour aborder les aspects passés, actuels et futurs des événements traumatiques (Shapiro, 2001). Pendant les séances EMDR, les patients se concentrent sur un souvenir perturbant en subissant simultanément une stimulation bilatérale. La thérapie EMDR individuelle a été jugée efficace pour traiter l'ESPT : elle entraîne une réduction significative des symptômes dans plus d'une douzaine d'essais contrôlés randomisés et de nombreuses méta-analyses (par exemple, Bisson, Roberts, Andrew, Cooper et Lewis, 2013 ; Watts et coll., 2013). On se reportera aux programmes d'aide humanitaire EMDR (HAP, 2016) pour un résumé des résultats de recherche.

La thérapie EMDR peut également être utilisée en groupe pour des patients ayant subi le même type de traumatismes (p. ex. abus sexuels, violences interpersonnelles graves), le même type d'expérience (p. ex. victimes de catastrophes naturelles, réfugiés, victimes de guerre), ou ayant des passés traumatiques variés, mais présentant des caractéristiques communes (p. ex. maladie chronique, maladie grave, violence domestique). Le protocole EMDR intégratif de traitement de groupe (EMDR-PITG, Artigas, Jarero, Alcalá \& López Cano, 2014) emploie les huit phases du traitement EMDR individuel (Shapiro, 2001) pour le traitement d'un groupe de patients par le biais d'un élément d'art-thérapie (utilisation de dessins) et du "Butterfly Hug » (BH ; Artigas \& Jarero, 2014). Le BH est une méthode de stimulation bilatérale autoadministrée 
permettant le traitement du matériel traumatique. L'EMDR-PITG contient toutes les composantes du traitement EMDR individuel, à l'exception des éléments cognitifs des phases 3 et 5 , ceux-ci étant trop complexes à gérer en groupe : l'installation cognitive de la phase 5 du traitement EMDR individuel est remplacée dans le protocole EMDR-PITG par la procédure de vision de l'avenir (décrite plus loin). L'efficacité de l'EMDR-IGTP a été documentée pour des groupes grands et petits de participants, adultes et enfants, avec nombre de rapports de cas et d'études de terrain, ainsi que neuf essais randomisés. On se reportera à Jarero, Artigas, Uribe \& Miranda (2014) pour une revue des recherches associées. Le présent article traite de l'adaptation du protocole aux patients adultes subissant le stress continu du diagnostic et du traitement du cancer (voir tableau 1).

\section{La thérapie EMDR administrée aux patients atteints du cancer}

Format individuel. Dans une étude pilote menée par Capezzani et coll. (2013) sur des patients atteints du cancer et souffrant d'ESPT, on administrait un traitement EMDR individuel à des patients en phase active du traitement du cancer $(n=10)$ et des patients assignés au hasard au traitement EMDR $(\mathrm{n}=11)$ ou à la TCC $(\mathrm{N}=10)$ lors de la phase de suivi du traitement du cancer. L'efficacité du traitement EMDR individuel (dans les phases actives et de suivi du traitement du cancer) s'est vue démontrée par les baisses significatives des notes à l'Échelle révisée d'impact de l'événement (IES-R) et à l'Échelle d'ESPT administrée par les cliniciens. Le traitement TCC, dans cette étude, montrait une efficacité limitée, et l'EMDR produisait des notes nettement inférieures à celles de la TCC aux deux échelles. Dix des onze patients traités avec l'EMDR n'avaient plus de diagnostic d'ESPT après traitement, contre un seul des patients traités par la TCC.

Format de groupe. Jarero et coll. (2015) ont mené une étude pilote afin d'évaluer l'EMDR-IGTP adapté aux adolescents et aux adultes vivant un stress traumatique continu, et son efficacité à réduire les états de stress lié au cancer chez des femmes adultes. La thérapie intensive EMDR-IGTP a été administrée pendant trois jours consécutifs, deux fois par jour, à 24 femmes adultes ayant différents types de cancer (du col de l'utérus, du sein, du côlon, de la vessie et de la peau) et présentant des symptômes d'ESPT liés à l'annonce de ce diagnostic et à leur traitement oncologique. On a comparé les résultats entre les patientes en phase active du traitement contre le cancer et celles en phase de suivi, avec le relevé des notes à l'Entretien bref d'évaluation de l'ESPT (SPRINT ; Connor $\&$ Davidson, 2001) en pré et post-traitement EMDR, et à 30 et 90 jours de suivi. Les résultats n'ont montré aucune différence entre les groupes, avec une amélioration significative dans les deux groupes des symptômes d'ESPT et du bien-être subjectif général.

\section{Le protocole de traitement de groupe intégratif EMDR adapté aux adolescents et aux adultes vivant un stress traumatique permanent}

Vue générale

Une séance individuelle d'évaluation et de relevé d'historique est menée pour chaque candidat éventuel à lentrée dans le groupe afin de déterminer s'ils sont prêts et aptes à recevoir le traitement EMDR selon les procédures standard (Shapiro, 2001). Six séances de groupe sont ensuite menées sur trois jours consécutifs, avec une séance le matin et l'après-midi. Les phases 2 à 7 sont effectuées au cours de chacune de ces séances de groupe très structurées. Au cours de ce processus, les patients suivent les instructions du thérapeute et travaillent dans le calme et de manière indépendante sur leurs propres souvenirs traumatiques, en utilisant l'EMDR-IGTP adapté (Jarero et coll., 2015), comme décrit dans le tableau 1 . Si, au cours de la phase 8 et des recommandations de suivi, on détermine qu'un ou plusieurs patients sont toujours perturbés après toutes les séances de groupe, plusieurs séances EMDR, individuelles ou en groupe restreint (trois à huit participants), peuvent être administrées.

Le traitement est mené sous forme intensive (deux fois par jour pendant trois jours consécutifs), car beaucoup de patients vivent loin de l'hôpital et n'y restent que pour un temps limité ; ce format intensif leur permet de recevoir la totalité du traitement sur une courte période. Le traitement peut être administré à un grand nombre de patients simultanément dans la mesure où il n'y a pas d'interaction entre les membres du groupe, à l'exception de l'opportunité (rarement utilisée) d'un dialogue rapide lors de la clôture, pendant les dernières minutes de la séance. Dans l'EMDR-IGTP, le format de groupe permet d'administrer le traitement individuel à un groupe de patients, donnant la possibilité à de nombreuses personnes d'être traitées en même temps. C'est là un point précieux dans les situations où les ressources sont limitées.

La thérapie EMDR-PITG est très différente de la thérapie de groupe traditionnelle (par exemple, 
Phase 1 : historique et évaluation des patients

Une séance individuelle est conduite afin d'obtenir l'historique et l'évaluation du client selon les procédures EMDR standard (Shapiro, 2001) et dans le but de déterminer si le client est apte et prêt au traitement.

Notez que la séquence suivante des phases 2 à 8 est fournie à chaque séance.

Phase 2 : préparation

Dans la première séance de groupe, les patients apprennent la méthode du Butterfly Hug (BH) pour l'auto-administration des stimulations bilatérales (Artigas \& Jarero, 2014). Les patients sont également familiarisés avec l'échelle d'unités subjectives de perturbation (SUD) où 0 représente aucune perturbation et 10 une perturbation maximale (Shapiro, 2001). On leur indique encore comment diviser leur feuille en quatre quadrants, portant chacun une lettre : A, B, C et D. Toutes les séances de groupe suivantes commencent par des instructions sur une technique d'auto-apaisement. On se reportera à Jarero \& Artigas (2016) pour des instructions détaillées sur quelques exercices d'auto-apaisement.

Phase 3 : évaluation

Film mental. Lors de la première séance de groupe, le chef de groupe demande aux patients de fermer les yeux et de " visualiser un film mental de tout ce qui s'est passé juste avant l'incident initial (par exemple, un diagnostic de cancer ou d'une autre maladie chronique ou longue) et de suivre jusqu'au présent », puis d'identifier ensuite «le moment le plus difficile, le plus douloureux ou le plus alarmant » ainsi que les émotions et les sensations corporelles associées. Dans toutes les séances de groupe ultérieures, le chef de groupe demandera aux patients de visualiser leur film mental et de choisir, pour le retraiter, tout souvenir qui les perturbe sur le moment.

Phase 4 : désensibilisation

Image A. On demande aux patients de dessiner cette expérience dans le carré A et de donner ensuite une note SUD pour cette image. Le thérapeute fait faire aux patients le BH et leur dit de s'arrêter lorsqu'ils sont prêts (environ 2-3 minutes).

Image B. Le thérapeute demande ensuite aux patients de représenter par un dessin leur état intérieur dans le carré B, puis de fournir une nouvelle note SUD pour cette image. Il leur demande ensuite de faire le BH, s'arrêtant lorsqu'ils sont prêts (environ 2-3 minutes).

Images C et D. La procédure de l'image B est répétée pour chacune des images C et D.

Note SUD de fin de séance. Le thérapeute demande aux patients d'observer attentivement tous les dessins, puis de choisir celui qui les a le plus perturbés, de retourner ensuite leur feuille et d'écrire une nouvelle note SUD représentant leur niveau de perturbation présent.

Phase 5 : vision du futur

Image de vision du futur. Le thérapeute demande aux patients de dessiner la façon dont ils se voient dans le futur et de donner un titre à leur dessin par un mot ou une phrase. Ils font ensuite le $\mathrm{BH}$.

Phase 6 : scan corporel

Le thérapeute demande aux patients de se souvenir du dessin qui était le plus perturbant et d'observer leurs sensations physiques, agréables ou désagréables, puis de faire le $\mathrm{BH}$.

Phase 7 : clôture

Les patients effectuent l'exercice d'auto-apaisement avec lequel ils se sentent le plus à l'aise et on leur donne ensuite l'occasion de partager leur vécu du retraitement. Les membres de l'équipe de protection émotionnelle (EPE) normalisent ces expériences de retraitement, en expliquant qu’elles sont normales, et répondent aux questions.

Phase $8:$ recommandations de réévaluation et de suivi

À la fin de chaque séance de groupe, les membres de l'EPE examinent le matériel de chaque patient afin d'identifier toute personne ayant besoin d'une assistance supplémentaire (voir l'exemple clinique suivant). À la fin de toutes les séances de groupe, les patients nécessitant plus de traitement sont identifiés par l'équipe.

Note. Ce texte a été tiré de Jarero \& Artigas (2016).

Yalom, 1970) qui favorise l'interaction entre les membres du groupe qui s'apportent soutien et validation mutuels, et dans lequel le processus de groupe est considéré comme un des principaux mécanismes de changement. Elle est également très différente de la TCC de groupe centrée sur le traumatisme (TCC-FT), dans la mesure où les composantes de la TCC-FT comprennent l'élaboration d'un récit entourant le trauma 
ainsi qu'une exposition graduelle in vivo (Deblinger \& Pollio, 2013). Dans le traitement EMDR-IGTP, l'entretien individuel initial d'admission est la seule occasion qu'ont les patients de raconter brièvement leur histoire et d'exprimer verbalement leur détresse. Une autre différence importante est la confidentialité. Dans le traitement EMDR-IGTP, la confidentialité est stricte. Comme l'ont noté Deblinger, Pollio et Dorsey (2016) en référence à la TCC-FT en groupe, "les questions de confidentialité sont traitées de la même manière que pour la thérapie individuelle ; toutefois, bien que les membres du groupe soient encouragés à maintenir la confidentialité, il est important de noter que le respect d'une confidentialité stricte par les autres membres du groupe ne peut être garantie » (p. 62).

Le groupe de thérapeutes EMDR-IGTP comprend le chef d'équipe et les membres de l'équipe de protection émotionnelle (EPE). Nous recommandons un ratio d'un membre de l'EPE pour 8 à 10 patients. Les membres de l'EPE circulent parmi les patients afin qu'ils puissent intervenir avant, pendant et après la procédure de groupe. Si un patient se sent en détresse au cours de la procédure, un membre de l'EPE s'approche discrètement de lui pour montrer son soutien et, si nécessaire, lui proposer un mouchoir. Tout cela se passe en silence, et il n'y a pas d'interaction entre le patient et le membre de l'EPE, afin de ne pas déranger les autres patients qui travaillent sur leur propre matériau. Les patients peuvent également partager des informations ou poser des questions au membre de l'EPE avant et après la procédure.

\section{Modification de I'EMDR-IGTP}

Dans l'EMDR-IGTP standard, le chef d'équipe demande aux patients de se " remémorer ce qui s'est passé pendant l'événement » (p. ex. un ouragan, un séisme). Au lieu de cela, dans cette adaptation pour le stress traumatique continu, le chef d'équipe demande aux patients de "visualiser un film mental de tout ce qui s'est passé juste avant l'incident initial (p. ex. le diagnostic du cancer ou d'une autre maladie chronique) jusqu'à maintenant ». L'événement cible initial du traitement sera le moment le plus perturbant du film mental. Au cours des séances suivantes, le chef d'équipe demande aux patients de repasser leur film mental et de cibler tout souvenir perturbant qui se présente, en remarquant les émotions et les sensations corporelles associées (voir le tableau 1 pour le détail des instructions).

Cette adaptation de l'EMDR-IGTP standard a été proposée car, selon nous, l'expérience traumatique que représente le cancer n’est pas un événement isolé suivi d'une période de sécurité post-traumatique, mais une expérience marquée par des traumatismes répétés et d'une durée indéterminée. Par conséquent, l'identification d'un facteur de stress spécifique parmi les crises multiples que constitue l'expérience du cancer est plus difficile que pour d'autres types de traumas. La modification du protocole permet de cibler et de retraiter tous les incidents connexes.

\section{Exemple clinique}

\section{La patiente}

Eva était une jeune femme de 32 ans, divorcée, vivant avec ses deux enfants, âgés de 7 et 9 ans. Elle habitait une petite ville située à près d'une journée de route de l'hôpital. Au moment de cette intervention, elle résidait dans un refuge de la Pink Cross. Elle était traitée pour un cancer du sein non métastatique. Elle avait déjà eu une mastectomie et était maintenant sous chimiothérapie.

\section{Évaluation}

Eva présentait des symptômes sévères d'ESPT. Lors de son évaluation individuelle, elle décrivait les symptômes suivants. Ses cognitions négatives étaient « Je suis différente », "Je suis laide parce que mon corps est dégoûtant » et "Je suis insignifiante », avec des pensées répétitives telles que "Je suis mutilée » et « Il me reste peu de temps à vivre ». Elle rapportait de nombreux flash-backs et images intrusives : (a) après la mastectomie, voyant son corps mutilé dans le miroir et réalisant qu'elle n'avait plus qu'un sein ; (b) la première fois qu'elle avait reçu une chimiothérapie ; et (c) le regard apitoyé de ses amis. Elle rapportait également une intuition douloureuse du futur, s'imaginant en train de mourir seule : "Personne ne s'occupera de moi ». Eva était perturbée par des cauchemars dans lesquels le cancer revenait et où elle devait avoir sans cesse des chimiothérapies. Elle disait se sentir très triste, désespérée, angoissée et impuissante, et elle pleurait sans pouvoir s'arrêter. Elle évitait les gens, ayant l'impression d'être un objet de curiosité morbide, et elle s'était coupée de tout le monde, même de ceux qu'elle aimait. Elle pensait que sa maladie était « un châtiment de Dieu à cause de ses péchés » et "qu'elle n'irait pas au paradis".

Eva présentait une note au SPRINT de 18 en prétraitement (Connor \& Davidson, 2001), une note supérieure au seuil clinique de 14 qui reflétait bien sa détresse. Le SPRINT a de solides propriétés psychométriques ; c'est un questionnaire composé de huit items, passé sous forme d'entretien ou en 
auto-évaluation. Des notes comprises entre 18 et 32 correspondent à des symptômes d'ESPT marqués ou graves, entre 11 et 17 à des symptômes modérés, entre 7 et 10 à des symptômes légers, et 6 ou moins à des symptômes minimes ou inexistants.

\section{Réponse au traitement}

Séance 1 (matinée $d u$ premier jour). Au cours de la première séance de traitement, Eva dessina la séquence d'images suivante : (a) un visage en noir en train de pleurer (échelle d'unités subjectives de perturbation $[\mathrm{SUD}]=10)$, (b) un visage triste en bleu (SUD = 8), (c) un visage heureux en vert (SUD $=5$ ), et (d) un visage en noir (SUD = 6). Au dos de la feuille, une note SUD de fin de séance de 7 et pour la vision de l'avenir : le dessin d'un trou noir, titré « Il n'y a pas d'espoir pour nous " (cf. figure 1).

Une partie des tâches des membres de l'EPE pendant le protocole de groupe est d'être attentifs aux
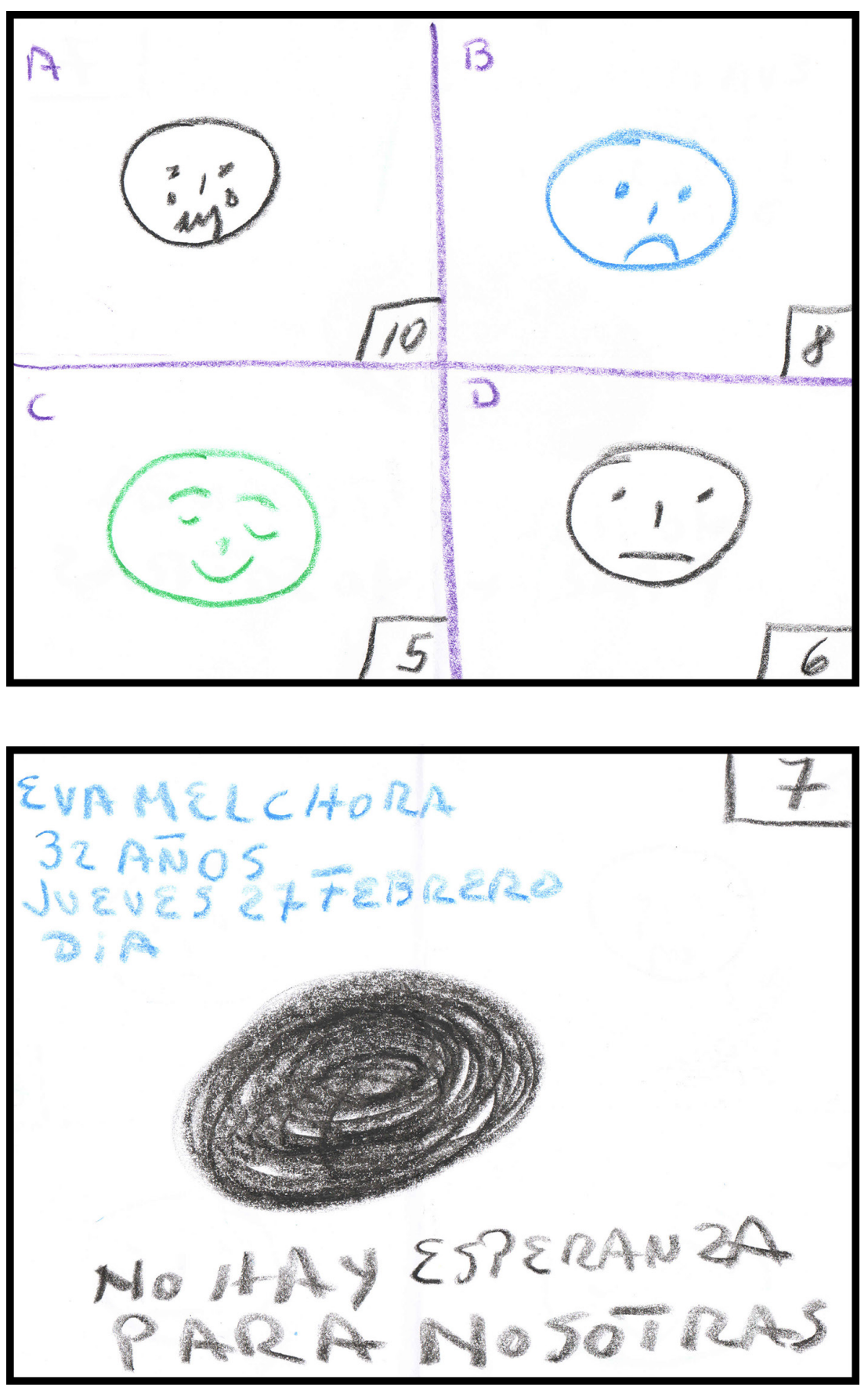

FIGURE 1. Dessin d'Eva, séance 1. En haut : 4 images traitées au cours de la séance. En bas : vision de l'avenir, un trou noir avec pour titre : « Pas d'espoir pour nous » (SUD = 7). 
réactions du patient (principalement pour lui fournir des mouchoirs, mais sans interagir verbalement avec lui), aux dessins, aux notes de SUD et aux titres des dessins de vision de l'avenir. Dans ce cas particulier, Eva ne pleurait pas ; par conséquent, le membre de l'EPE avait simplement pris note d'Eva et de sa page de vision de l'avenir. Au cours de la procédure de réévaluation de la phase 8 , tenue par l'équipe EPE immédiatement après chaque séance, au moment où les patients quittent la salle, les membres de l'équipe examinent attentivement chaque dessin, les notes SUD et les titres des dessins de vision de l'avenir, et ils écoutent également les rapports de chaque membre de l'EPE. Dans ce cas particulier, le membre de l'EPE mentionna le cas d'Eva. L'EPE décida qu'avant le retraitement de groupe suivant, ce membre de l'EPE devrait demander à Eva, en privé, d'apporter une modification mineure lorsque le leader donnerait au groupe les instructions concernant le film mental (phase 3). On lui demanderait d'observer la consigne suivante : "Déroulez un film mental d'aujourd'hui vers l'avenir... et lorsque vous avez fini, choisissez de retravailler sur ce qui vous perturbe sur le moment. »

Séance 2 (après-midi du premier jour). Au cours de la deuxième séance de traitement des traumas, Eva dessina la séquence d'images suivante : (a) une spirale noire (SUD $=8)$, (b) un visage vert triste (SUD = 5), (c) un visage bleu agréable (SUD $=3$ ), et (d) un visage violet heureux (SUD = 2). Au dos du document, une note SUD de fin de séance de 4 et un dessin de vision de l'avenir représentant le symbole chrétien du poisson. Le titre du dessin était «J’ai foi en Jésus » (voir la figure 2).

Séance 6 (après-midi du troisième jour). Au cours de la séance finale, Eva dessina la séquence d'images suivante : (a) un visage vert heureux ( $\mathrm{SUD}=3$ ), (b) un visage orange heureux (SUD $=4$ ), (c) un visage bleu heureux $(S U D=2)$ et $(D)$ un visage rouge heureux (SUD = 1). Au dos du document, une note SUD de fin de séance de 1. Sa vision de l'avenir était représentée par un soleil et portait le titre : "Je souhaite partager mon bonheur» (voir la figure 2).

\section{Post-traitement et suivi}

La note au SPRINT d'Eva, de 18 en prétraitement (Connor \& Davidson, 2001), indiquait une intensité sévère et dépassait le seuil clinique de 14 . En post-traitement, sa note était descendue à 9 , dans la gamme des symptômes légers. Lors du suivi à 30 jours, cette note était de 5 et de 4 à 90 jours (les deux indiquant des symptômes minimes ou inexistants). Il est important de noter que cette note s'était maintenue alors
qu'Eva était toujours en traitement actif du cancer du sein, qu'elle recevait toujours de la chimiothérapie et se rendait à diverses analyses de suivi et d'évaluations.

\section{Discussion}

Cet article fournit une description détaillée de l'EMDR-IGTP adapté aux adolescents (de 13 à 17 ans) et aux adultes vivant un stress traumatique continu. Comme indiqué précédemment, cette procédure a été jugée efficace dans une étude pilote portant sur des patients atteints du cancer (Jarero et coll., 2015). Ce protocole est une modification de l'EMDR-IGTP, une procédure qui a été utilisée avec succès dans le monde entier pour fournir une réponse rapide à de grands groupes de participants à la suite de catastrophes (Jarero \& Artigas, 2016). L'adaptation touche la façon dont le souvenir ciblé est sélectionné ; elle a été faite pour permettre le traitement du continuum d'incidents traumatiques multiples auxquels font face les clients confrontés à un stress traumatique continu.

Il est important de mentionner que l'EMDR-PITG n'exclut pas un traitement EMDR individuel, car c'est un protocole thérapeutique, mais aussi un outil de dépistage. Par conséquent, les cliniciens EMDR n’ont pas à choisir entre une utilisation en groupe ou une utilisation individuelle du protocole lorsqu'ils font face à un grand nombre de personnes à traiter. Nous recommandons d'utiliser d'abord l'EMDR-IGTP et de n'administrer un traitement EMDR individuel qu'à ceux qui ont besoin d'un soutien supplémentaire, en fonction des recommandations de la phase 8 (réévaluation et suivi) du protocole.

\section{Indications et contre-indications pour le traitement}

Nous utilisons les critères de sélection décrits dans l'ouvrage de Shapiro (2001). De plus, nous avons signalé l'importance du soutien social. Une des leçons les plus importantes pour nous, au cours de ces recherches auprès de patientes atteints de cancer, a été de constater que la différence principale entre le reste du groupe et les deux patientes ayant besoin d'une thérapie individuelle après l'EMDR-IGTP était l'absence complète de facteurs structurels (sociaux) et fonctionnels (familiaux) autour de ces deux patientes. Nous avons depuis lors ajouté « manque complet de soutien structurel (social) et fonctionnel (familial) » à nos critères d'exclusion.

\section{Tolérance au traitement}

Au cours de nos dix-huit années de travail sur le terrain avec l'EMDR-IGTP, nous n'avons jamais vu un 

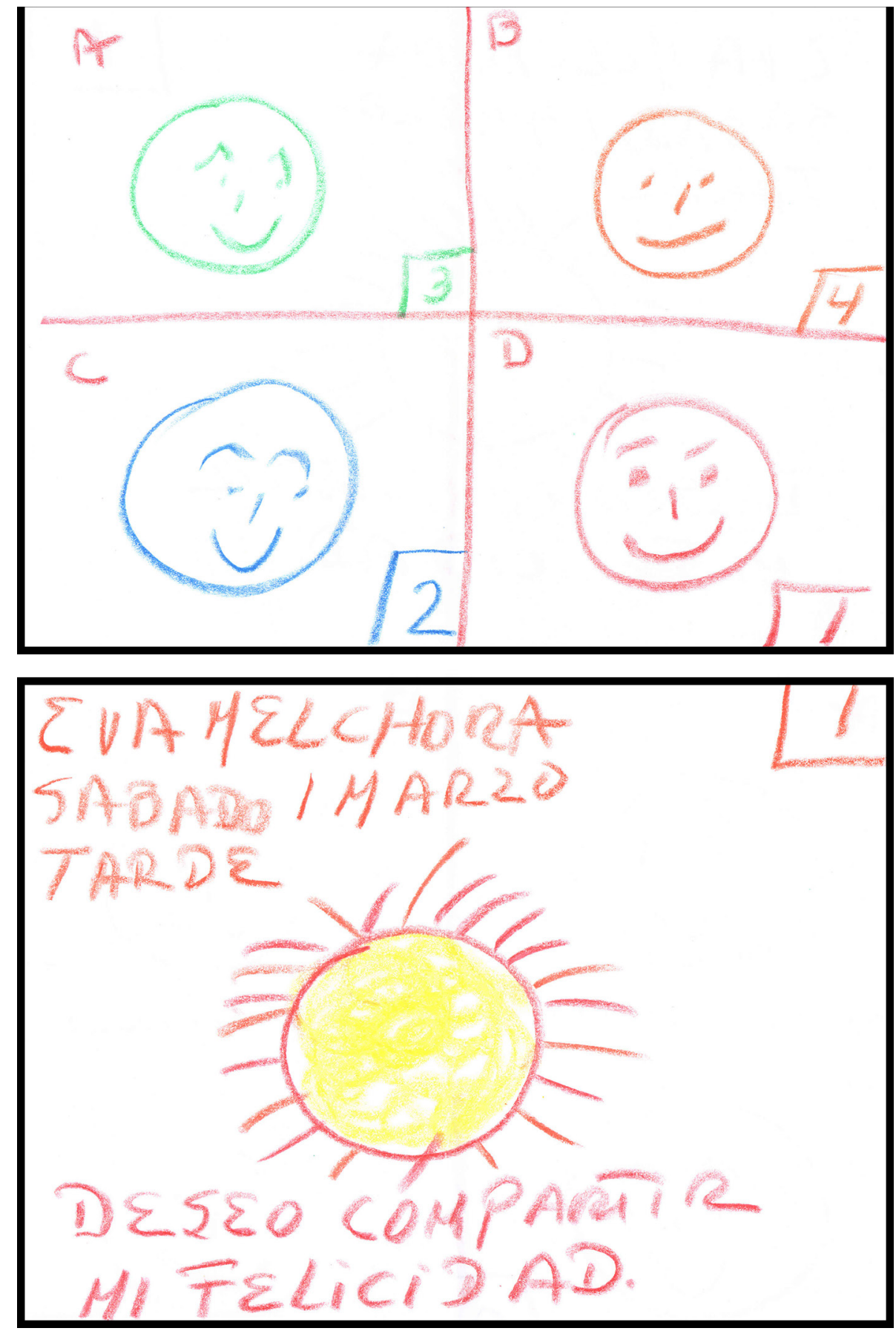

FIGURE 2. Dessin d'Eva, séance 6. En haut : 4 images traitées en séance. En bas (vision de l'avenir) : un soleil avec comme titre : «Je veux partager mon bonheur » $(\mathrm{SUD}=1)$.

patient quitter la salle parce qu'il était trop perturbé. Cela tient peut-être au fait que nous appliquons avec beaucoup d'attention nos critères de sélection. Cependant, nous supposons aussi que l'utilisation de la méthode $\mathrm{BH}$ comme stimulation bilatérale (Artigas et Jarero, 2014) maintient les patients au sein de leur fenêtre de tolérance et permet un retraitement approprié. Il se peut également que le $\mathrm{BH}$ fournisse aux clients un sentiment de contrôle sur leur traitement et leur permette de préserver un sentiment de sécurité lors du traitement de souvenirs traumatiques.

\section{Recommandations}

À l'appui de cette pratique en psycho-oncologie, le premier principe de la constitution de l'OMS (2006) donne la priorité à la contribution du bien-être total au bonheur, aux relations harmonieuses et à la sécurité 
de toutes les personnes, affirmant que " la santé est un état de bien-être physique, mental et social complet, et pas seulement l'absence de maladie ou d'infirmité ». Ce qui signifie qu'il n'y a pas de santé sans santé mentale (Prince et coll., 2007). La commission cancer du Collège américain de chirurgie a fait du dépistage de la détresse psychosociale une nouvelle norme de soins pour les patients en 2015. Cette norme nécessite des programmes accrédités afin de mettre en place un protocole systématique de dépistage des perturbations émotionnelles chez tous les patients atteints de cancer et d'offrir un suivi adapté et une orientation vers des professionnels qualifiés lorsque cela s'avère nécessaire (Kendall, 2015).

La recherche et la pratique en psycho-oncologie ont joué un rôle-clé dans la réduction du risque de cancer ; elles ont amélioré le taux de survie des malades et produit des changements sociaux et culturels réduisant la stigmatisation liée à la maladie (Dunn, Adams, Holland et Watson, 2015). En conséquence, nous recommandons d'autres recherches sur le protocole EMDR-IGTP adapté, avec des études randomisées contrôlées, suivant les recommandations méthodologiques de Maxfield et Hyer (2002).

\section{Bibliographie}

American Psychiatric Association. (2013). Diagnostic and statistical manual of mental disorders (5th ed.). Washington, DC: Author.

Andrykowski, M., \& Kangas, M. (2010). Posttraumatic stress disorder associated with cancer diagnosis and treatment. In J. C. Holland, W. S. Breitbart, P. B. Jacobsen, M. S. Lederberg, M. J. Loscalzo, \& R. McCorklen (Eds.), Psycho-oncology (2nd ed., pp. 348-357). New York, NY: Oxford University Press.

Artigas, L., \& Jarero, I. (2014). The butterfly hug. In M. Luber (Ed.). Implementing EMDR early mental health interventions for man-made and natural disasters: Models, scripted protocols, and summary sheets (pp. 127-131). New York, NY: Springer Publishing.

Artigas, L., Jarero, I., Alcalá, N., \& López Cano, T. (2014). The EMDR Integrative Group Treatment Protocol (IGTP) for Children. In M. Luber (Ed.). Implementing EMDR early mental health interventions for man-made and natural disasters: Models, scripted protocols, and summary sheets (pp. 237251). New York, NY: Springer Publishing.

Bisson, J., Roberts, N. P., Andrew, M., Cooper, R., \& Lewis, C. (2013). Psychological therapies for chronic posttraumatic stress disorder (PTSD) in adults. Cochrane Database of Systematic Reviews, (12), CD003388. http:// dx.doi. org/10.1002/14651858.CD003388.pub4

Capezzani, L., Ostacoli, L., Cavallo, M., Carletto, S., Fernandez, I., Solomon, R.,. . . Cantelmi, T. (2013). EMDR and CBT for cancer patients: Comparative study of effects on PTSD, anxiety, and depression. Journal of EMDR Practice and Research, 7(3), 134-143.

Connor, K. M., \& Davidson, J. R. (2001). SPRINT: A brief global assessment of post-traumatic stress disorder. International Clinical Psychopharmacology, 16(5), 279-284.

Deblinger, E., \& Pollio, E. (2013). Implementing trauma focused cognitive behavioral therapy (TF-CBT) in a group format. Récupéré à l'adresse http://www.cpe.rutgers. edu / njdcf2013 / docs / 008-Implementing-Trauma-Focused-Cognitive-Behavioral-Therapy-in-a-Group-Format.pdf

Deblinger, E., Pollio, E., \& Dorsey, S. (2016). Applying trauma-focused cognitive-behavioral therapy in group format. Child Maltreatment, 21(1), 59-73.

Dunn, J., Adams, C., Holland, J., \& Watson, M. (2015). Reinforcing the role of psycho-social oncology in global cancer prevention: Applying psycho-oncology research in programmes and practice. Psycho-Oncology, 24, 1217-1221.

EMDR Humanitarian Assistance Programs. (2016). Trauma recovery EMDR Humanitarian Assistance Programs. Research findings. Récupéré à l'adresse http://www. emdrhap.org/content/what-is-emdr/research-findings

Jarero, I., Amaya, C., Givaudan, M., \& Miranda, A. (2013). EMDR Individual protocol for paraprofessional use: A randomized controlled trial with first responders. Journal of EMDR Practice and Research, 7(2), 55-64.

Jarero, I., \& Artigas, L. (2016). EMDR integrative group treatment protocol for adolescents (14-17 years) and adults living with ongoing traumatic stress. In $\mathrm{M}$. Luber (Ed.). Eye movement desensitization and reprocessing (EMDR) therapy scripted protocols and summary sheets: Treating trauma- and stressor-related conditions (pp. 169180). New York, NY: Springer Publishing.

Jarero, I., Artigas, L., \& Luber, M. (2011). The EMDR protocol for recent critical incidents: Application in a disaster mental health continuum of care context. Journal of EMDR Practice and Research, 5(3), 82-94.

Jarero, I., Artigas, L., Uribe, S., García, L. E., Cavazos, M. A., \& Givaudan, M. (2015). Pilot research study on the provision of the EMDR integrative group treatment protocol with female cancer patients. Journal of EMDR Practice and Research, 9(2), 98-105.

Jarero, I., Artigas, L., Uribe, S., \& Miranda, A. (2014). EMDR therapy humanitarian trauma recovery interventions in Latin America and the Caribbean. Journal of EMDR Practice and Research, 8(4), 260-268.

Jarero, I., \& Uribe, S. (2011). The EMDR protocol for recent critical incidents: Brief report of an application in a human massacre situation. Journal of EMDR Practice and Research, 5(4), 156-165.

Jarero, I., \& Uribe, S. (2012). The EMDR protocol for recent critical incidents: Follow-up report of an application in a human massacre situation. Journal of EMDR Practice and Research, 6(2), 50-61. 
Kangas, M., Henry, J. L., \& Bryant, R. A. (2002). Posttraumatic stress disorder following cancer: A conceptual and empirical review. Clinical Psychology Review, 22, 499-524.

Kangas, M., Henry, J. L., \& Bryant, R. A. (2007). Correlates of acute stress disorder in cancer patients. Journal of Traumatic Stress, 20(3), 325-334.

Kendall, J. (2015). Distress screening from paper/pencil to EHR integration. Psycho-Oncology, 24, 6. http://dx.doi .org/ $10.1002 /$ pon.3873

Logie, R., \& De Jongh, A. (2014). The "flashforward" procedure: Confronting the catastrophe. Journal of EMDR Practice and Research, 8, 25-32.

Maxfield, L., \& Hyer, L. (2002). The relationship between efficacy and methodology in studies investigating EMDR treatment of PTSD. Journal of Clinical Psychology, 58, 23-41.

Muszbek, K. (2010). Anxiety and adjustment disorders in cancer patients. IPOS online curriculum. Récupéré à l'adresse : http://docs.ipos-society.org/education/ core_curriculum/en/Muszbek_anx/player.html

National Cancer Institute. (2015). Adjustment to cancer: Anxiety and distress (PDQ (B). Récupéré à l'adresse http:/ / www.cancer.gov/about-cancer/ coping/feelings / anxiety-distress-pdq\#section/_56
Organisation mondiale de la santé. (2006). Constitution de l'Organisation mondiale de la santé. Genève, Suisse : Auteur.

Organisation mondiale de la santé. (2013). Évaluation et prise en charge des affections spécifiquement liées au stress. Genève, Suisse : Auteur.

Prince, M., Patel, V., Saxena, S., Maj, M., Maselko, J., Phillips, M. R., \& Rahman, A. (2007). No health without mental health. Lancet, 370, 859-877.

Shapiro, F. (2001). Eye movement desensitization and reprocessing. Basic principles, protocols, and procedures (2nd ed.). New York, NY: Guilford Press.

Watts, B. V., Schnurr, P. P., Mayo, L., Young-Xu, Y., Weeks, W. B., \& Friedman, M. J. (2013). Meta-analysis of the efficacy of treatments for posttraumatic stress disorder. Journal of Clinical Psychiatry, 74(6), e541-e550. http:/ / dx.doi.org/10.4088/JCP.12r08225

Yalom, I. D. (1970). The theory and practice of group psychotherapy. New York, NY: Basic Books.

Merci d'adresser toute correspondance concernant cet article à Ignacio Jarero, Latin American \& Caribbean Foundation for Psychological Trauma Research, Mexico City, Mexico 01900, Mexique. Courriel : nacho@amamecrisis. com.mx 